\title{
High-resolution radio continuum survey of M 33
}

\section{Thermal and nonthermal emission}

\author{
F. S. Tabatabaei ${ }^{1, \star}$, R. Beck ${ }^{1}$, E. Krügel ${ }^{1}$, M. Krause ${ }^{1}$, E. M. Berkhuijsen ${ }^{1}$, K. D. Gordon ${ }^{2}$, and K. M. Menten ${ }^{1}$ \\ 1 Max-Planck Institut für Radioastronomie, Auf dem Hügel 69, 53121 Bonn, Germany \\ e-mail: tabataba@mpifr-bonn.mpg.de \\ 2 Steward Observatory, University of Arizona, 933 North Cherry Avenue, Tucson, AZ 85721, USA \\ Received 27 June 2007 / Accepted 10 August 2007
}

\section{ABSTRACT}

\begin{abstract}
Context. Constraints on the origin and propagation of cosmic rays can be achieved by studying the variation in the spectral index of the synchrotron emission across external galaxies.

Aims. We determine the variation in the nonthermal radio spectral index in the nearby spiral galaxy M 33 at a linear resolution of 360 pc.

Methods. We separated the thermal and nonthermal components of the radio continuum emission without the assumption of a constant nonthermal spectral index. Using the Spitzer FIR data at 70 and $160 \mu \mathrm{m}$ and a standard dust model, we dereddened the $\mathrm{H} \alpha$ emission. The extinction corrected $\mathrm{H} \alpha$ emission serves as a template for the thermal free-free radio emission. Subtracting this free-free emission from the observed $3.6 \mathrm{~cm}$ and $20 \mathrm{~cm}$ emission (Effelsberg and the VLA), we obtained the nonthermal maps. A constant electron temperature used to obtain the thermal radio intensity seems appropriate for M 33, which, unlike the Milky Way, has a shallow metallicity gradient.

Results. For the first time, we derive the distribution of the nonthermal spectral index across a galaxy, M33. We detect strong nonthermal emission from the spiral arms and starforming regions. Wavelet analysis shows that at $3.6 \mathrm{~cm}$ the nonthermal emission is dominated by contributions from starforming regions, while it is smoothly distributed at $20 \mathrm{~cm}$. For the whole galaxy, we obtain thermal fractions of $51 \%$ and $18 \%$ at $3.6 \mathrm{~cm}$ and $20 \mathrm{~cm}$, respectively. The thermal emission is slightly stronger in the southern than in the northern half of the galaxy. We find a clear radial gradient of mean extinction in the galactic plane.

Conclusions. The nonthermal spectral index map indicates that the relativistic electrons suffer energy loss when diffusing from their origin in starforming regions towards interarm regions and the outer parts of the galaxy. We also conclude that the radio emission is mostly nonthermal at $R>5 \mathrm{kpc}$ in $\mathrm{M} 33$.
\end{abstract}

Key words. galaxies: individual: M 33 - radio continuum: galaxies - galaxies: ISM - ISM: dust, extinction - ISM: cosmic rays

\section{Introduction}

The problem of separating the two components of the radio continuum emission, free-free (thermal) and synchrotron (nonthermal) emission, dates back to the beginnings of radioastronomy. The usually applied techniques are based on either assuming a constant nonthermal spectral index (e.g. Klein et al. 1984) or a correlation between thermal radio and infra-red (IR) emission (Broadbent et al. 1989).

Although the assumption of constant nonthermal spectral index may be reasonable for global studies (Sect. 10), it does not lead to a feasible thermal/nonthermal distribution in detailed studies. Under this simplification, it is not possible to investigate the origin and energy-loss processes of the electron component of cosmic rays (CRs) within a galaxy. Discrete synchrotronemitting sources are mainly identified as supernova remnants. The synchrotron emission from supernova remnants can be described as power law $\left(S_{v} \sim v^{-\alpha_{n}}\right)$ with a typical spectral index of $\alpha_{n} \simeq 0.6$. Propagating from these sources, electrons suffer energy losses that steepen the power-law spectrum. In the interstellar medium, the typical spectral index attributed to the emission under the leakage loss is $\simeq 0.9$ and $\simeq 1.1$ under synchrotron loss

* Member of the International Max Planck Research School (IMPRS) for Radio and Infrared Astronomy at the Universities of Bonn and Cologne. and inverse Compton scattering (Biermann 1995). These electrons diffuse further to the interarm regions and outer parts of spiral galaxies within their life time. Hence, variations in the nonthermal spectral index should be distinguishable particularly by comparing arms with interarm regions and outer parts of the galaxies.

The thermal/nonthermal separation based on the assumption that the radio-IR correlation is due to a correlation between the thermal radio and IR emission is not generally correct, because, nonthermal phenomena, e.g. super massive black holes or energetic CRs, may stimulate the IR emission. For instance, the possibility of heating the diffuse dust (emitting in IR) by CRs was shown by Helou \& Bicay (1993) and Bicay \& Helou (1990). Moreover, supernovae, the ultimate sources of most of the nonthermal emission, explode close to the starforming regions in which their proginator stars formed. Together with the increased magnetic field strength in the spiral arms, this causes a correlation between the thermal and nonthermal sources, making a less direct link between the IR and thermal emissions. Finally, the slope of the radio-IR correlation depends on the synchrotron spectral index (Niklas \& Beck 1997).

Templates for free-free emission could be provided by emission of recombination lines as they originate within ionized regions, like the free-free emission. The $\mathrm{H} \alpha$ emission, the strongest Balmer line, is most preferred observationally, particularly from nearby galaxies. Both the $\mathrm{H} \alpha$ and free-free 
Table 1. Images of M 33 used in this study.

\begin{tabular}{cccc}
\hline \hline @ noalign Wavelength & Resolution & rms noise & Telescope \\
\hline $20 \mathrm{~cm}$ & $51^{\prime \prime}$ & $70 \mu \mathrm{Jy} / \mathrm{beam}$ area & VLA+Effelsberg \\
$3.6 \mathrm{~cm}$ & $84^{\prime \prime}$ & $230 \mu \mathrm{Jy} / \mathrm{beam}^{1}$ area & Effelsberg $^{1}$ \\
$160 \mu \mathrm{m}$ & $40^{\prime \prime}$ & $10 \mu \mathrm{Jy} / \operatorname{arcsec}^{2}$ & Spitzer $^{2}$ \\
$70 \mu \mathrm{m}$ & $18^{\prime \prime}$ & $10 \mu \mathrm{Jy} / \mathrm{arcsec}^{2}$ & Spitzer $^{2}$ \\
$6570 \AA(\mathrm{H} \alpha)$ & $2^{\prime \prime}$ (pixel size) & $0.3 \mathrm{~cm}^{-6} \mathrm{pc}$ & KPNO $^{4}$ \\
\hline
\end{tabular}

${ }^{1}$ Tabatabaei et al. (2007a). ${ }^{2}$ Hinz et al. (2004) and Tabatabaei et al. (2007a). ${ }^{3}$ Hoopes \& Walterbos (2000).

emission are linearly proportional to the number of ionizing photons produced by massive stars. On the other hand, the $\mathrm{H} \alpha$ emission suffers from extinction by dust leading to an underestimate of the free-free emission if no extinction correction is made. Note that the emission of radio recombination lines is extinctionfree, hence ideal for tracing the free-free emission. However, these kinds of emission are too weak from the diffuse ionized gas in external galaxies to be detected using the present facilites. For example, our attempt to map radio recombination-line emission from diffuse regions within IC 342 and M 33 using the $6.2 \mathrm{~cm}$ receiver of the Effelsberg Telescope was not successful.

The nearest Scd galaxy, M33 (NGC 598), at a distance of $840 \mathrm{kpc}\left(1^{\prime \prime} \simeq 4 \mathrm{pc} \text {, Freedman et al. 1991) }\right)^{1}$ and a favorite inclination of $i=56^{\circ}$ (Regan \& Vogel 1994) has been extensively studied at radio and IR wavelengths. The central position of M 33 given by de Vaucouleurs \& Leach (1981) is $\operatorname{RA}(1950)=1^{\mathrm{h}} 31^{\mathrm{m}} 01.57^{\mathrm{s}}$ and $\operatorname{Dec}(1950)=30^{\circ} 24^{\prime} 15.0^{\prime \prime}$. The position angle of the major axis is $\mathrm{PA} \simeq 23^{\circ}$ (Deul \& van der Hulst 1987). So far, extinction studies in M33 have mostly focused on HII regions using either the Balmer (and Paschen) line ratios (e.g. Kwitter \& Aller 1981; Melnick et al. 1987; Petersen \& Gammelgaard 1997) or the ratio of $\mathrm{H} \alpha$ to radio emission (e.g. Israel \& Kennicutt 1980; Devereux et al. 1997). In the line ratio method, the emission from the diffuse ionized medium can hardly be detected in most of these recombination lines. The method based on the radio emission only works well when the thermal component of the radio continuum is independently known. For instance, the radio emission from single HII regions (and not HII complexes with a possible nonthermal emission contribution like NGC 604 and NGC 595, Dodorico 1978; Gordon et al. 1993) may be considered as the thermal emission.

Using the ISOPHOT 60 and $170 \mu \mathrm{m}$ data, Hippelein et al. (2003) found an anti-correlation between the flux density ratio of $\mathrm{H} \alpha / 60 \mu \mathrm{m}$ and $170 \mu \mathrm{m}$ flux density, suggesting the extinction to be related to the cold dust in M 33 .

We obtained the distribution of the dust optical depth at the $\mathrm{H} \alpha$ wavelength for the whole M 33 (an extinction map) by analyzing dust emission and absorption using the high sensitivity and resolution Multiband Imaging Photometer Spitzer (MIPS, Rieke et al. 2004) FIR data at 70 and $160 \mu \mathrm{m}$. This led to an $\mathrm{H} \alpha$ map corrected for extinction (de-extincted $\mathrm{H} \alpha$ map), our free-free template. The thermal and nonthermal maps at 3.6 and $20 \mathrm{~cm}$ were obtained at an angular resolution of 90" (equivalent to a linear resolution of $360 \mathrm{pc}$ ) by both the new (using a free-free template) and the standard methods (assuming a constant nonthermal spectral index), and the results are compared. Furthermore, we determine variations in the nonthermal spectral

1 Recent distance determinations by (Bonanos et al. 2006) using a detached eclipsing binary yield values between 730 and $964 \mathrm{kpc}$, in agreement with our assumption of $D=840 \mathrm{kpc}$. index across M33 as detected by the new method and discuss the exponential scale lengths of both thermal and nonthermal emission.

In Sect. 3 we derive the distribution of dust color temperature. This temperature and the $160 \mu \mathrm{m}$ flux density are used to obtain the dust optical depth (extinction) and its distribution in Sects. 4 and 5. We correct the $\mathrm{H} \alpha$ emission for the extinction and then convert it to the thermal radio emission, following Dickinson et al. (2003) (Sect. 6). The nonthermal intensity and spectral index maps are produced in Sects. 7 and 8. We discuss and compare the results from the new and standard methods in Sect. 9. Finally, conclusions are presented in Sect. 10.

\section{Data}

The radio continuum data at 3.6 and $20 \mathrm{~cm}$ are presented in Paper I (Tabatabaei et al. 2007b). At $3.6 \mathrm{~cm}$, M 33 was observed with the 100-m Effelsberg telescope of the MPIfR ${ }^{2}$. The $20 \mathrm{~cm}$ data were obtained from observations with the Very Large Array $\left(\mathrm{VLA}^{3}\right)$ corrected for missing spacing using the Effelsberg data at $20 \mathrm{~cm}$. We also used the latest combined epochs of MIPS Spitzer data at 70 and $160 \mu \mathrm{m}$ (as presented in Tabatabaei et al. 2007a, 2005). The H $\alpha$ map is from Kitt Peak National Observatory (KPNO) (Hoopes \& Walterbos 2000). Table 1 summarizes the data used in this work.

\section{Temperature distribution of dust}

We derived the color temperature of the dust, $T$, between $70 \mu \mathrm{m}$ and $160 \mu \mathrm{m}$ from the formula

$\frac{F_{1}}{F_{2}}=\frac{\nu_{1}^{\beta}}{\nu_{2}^{\beta}} \cdot \frac{B_{1}(T)}{B_{2}(T)}$

Here $F$ denotes the measured flux, $B(T)$ the Planck function, $v$ the frequency, and 1 and 2 refer to the wavelength of $70 \mu \mathrm{m}$ and $160 \mu \mathrm{m}$, respectively. The power-law index of the absorption efficiency at FIR-wavelengths, $\beta$, was set to 2 , which should be appropriate for interstellar grains (Andriesse 1974; Draine \& Lee 1984).

The MIPS FIR maps at 70 and $160 \mu \mathrm{m}$ were smoothed to an angular resolution of $40^{\prime \prime}$ and normalized to the same grid and center position. The resulting dust temperature distribution is shown in Fig. 1, indicating variations between 19 and

\footnotetext{
2 The 100-m telescope at Effelsberg is operated by the Max-PlanckInstitut für Radioastronomie (MPIfR) on behalf of the Max-PlanckGesellschaft.

3 The VLA is a facility of the National Radio Astronomy Observatory. The NRAO is operated by Associated Universities, Inc., under contract with the National Science Foundation.
} 


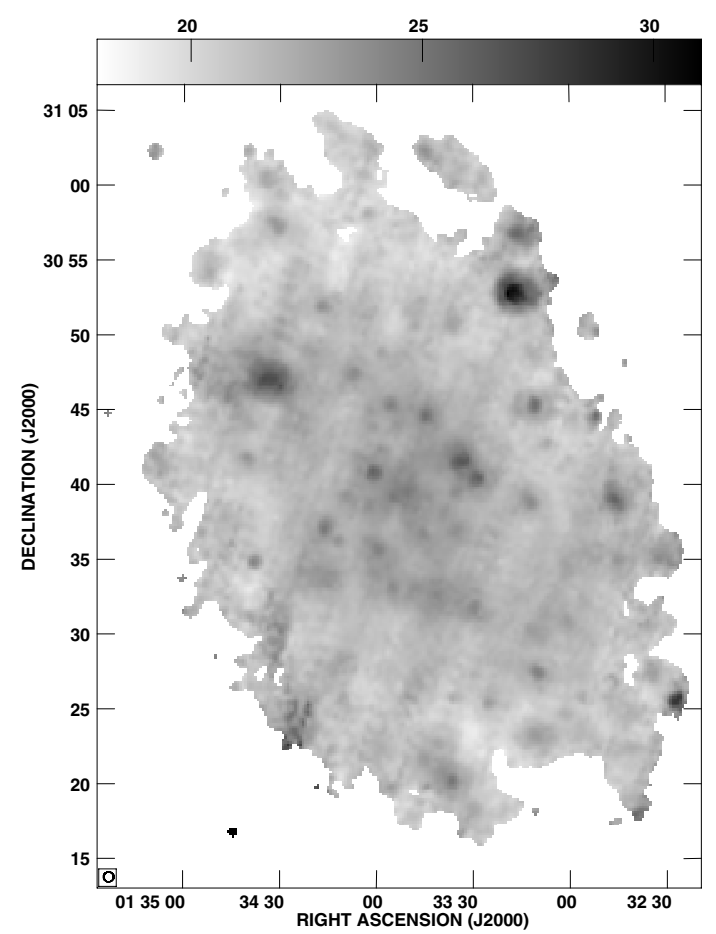

Fig. 1. Dust temperature map of M33 obtained from the $I_{70 \mu \mathrm{m}} / I_{160 \mu \mathrm{m}}$ ratio (only pixels with intensity above $3 \sigma$ level were used). The angular resolution of 40" is shown in the lower left corner of the map. The bar at the top gives the dust temperature in Kelvin.

$28 \mathrm{~K}$. Figure 2 displays the relative frequency of the temperature intervals in $10^{\prime \prime} \times 10^{\prime \prime}$ pixels. The maximum was attained at $21.5 \mathrm{~K}$, close to the mean value of $21.6 \mathrm{~K}$. Warmer dust with $T>25 \mathrm{~K}$ dominates in starforming regions and in the center of the galaxy. The highest temperatures are found in the HII complexes NGC 604, NGC 592, and IC 133.

\section{Optical depth at $160 \mu \mathrm{m}$}

Having determined $T$, we obtain the optical depth from the equation

$I_{160 \mu \mathrm{m}}=B_{160 \mu \mathrm{m}}(T)\left[1-\mathrm{e}^{-\tau_{160 \mu \mathrm{m}}}\right]$,

where $I_{160 \mu \mathrm{m}}$ is the intensity. The distribution of $\tau_{160 \mu \mathrm{m}}$ over the disk in M 33 is plotted in Fig. 3. Because the temperature variations are very moderate, the optical depth $\tau_{160 \mu} \mu$ usually follows the $160 \mu \mathrm{m}$ intensity map quite closely (see Tabatabaei et al. 2007a). Nevertheless, for a fixed intensity, a warm region (26 K) has a three times lower optical depth than a cold one (19 K).

\section{Distribution of extinction}

To convert $\tau_{160 \mu \mathrm{m}}$ into the dust optical depth at the wavelength of the $\mathrm{H} \alpha$ line, $\tau_{\mathrm{H} \alpha}$, we have to multiply it by $\kappa_{\mathrm{H} \alpha} / \kappa_{160 \mu \mathrm{m}}$, the ratio of the dust extinction coefficient per unit mass at the corresponding wavelengths. Using the extinction curve given by a standard dust model for the diffuse medium (see, e.g. Fig. 12.8 of Krügel 2003), we estimate $\tau_{\mathrm{H} \alpha} \simeq 2200 \tau_{160 \mu \mathrm{m}}$. Therefore, after multiplication with 2200, Fig. 3 also gives the distribution of $\tau_{\mathrm{H} \alpha}$ across the galaxy at a linear resolution of $360 \mathrm{pc}$.

In the extended central region and in the two main arms IN and IS, $\tau_{\mathrm{H} \alpha}$ is around 0.5 and somewhat smaller in other arms (mostly between 0.2 and 0.4 ). In the center of the galaxy and

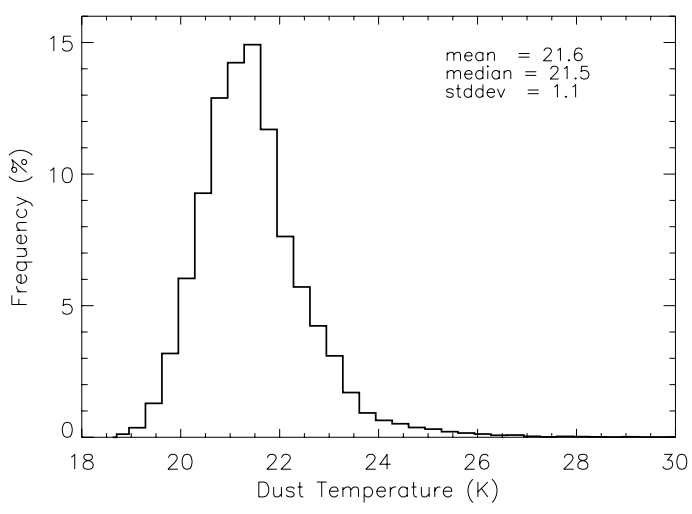

Fig. 2. Histogram of the dust temperature map. It shows the population of pixels as a function of the temperature intervals. The number of bins used for this plot is 80 .

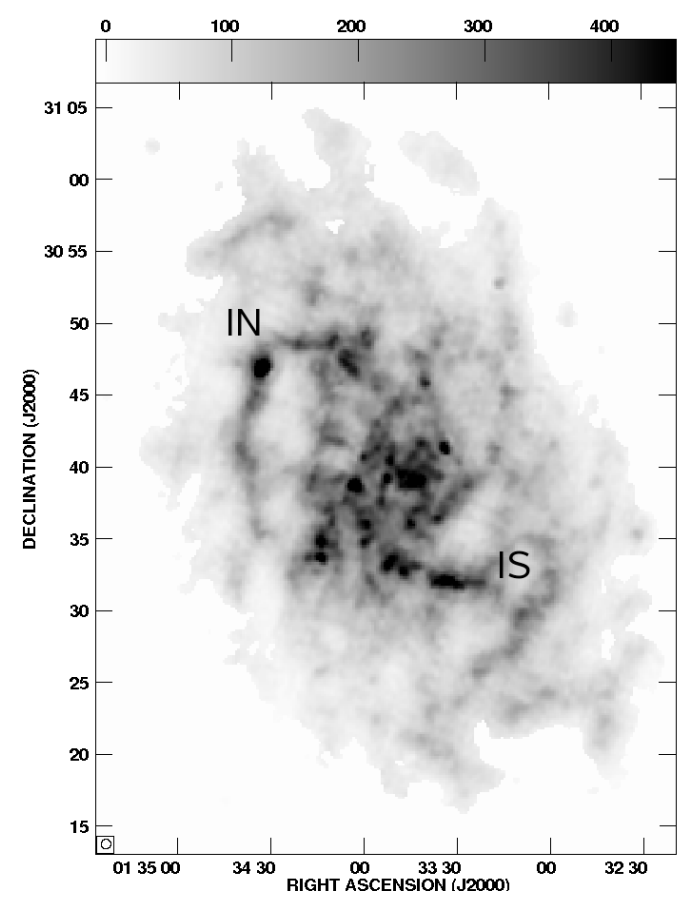

Fig. 3. Distribution of the dust optical depth at $160 \mu \mathrm{m}$ wavelength in M33. The bar at the top shows $10^{6} \times \tau_{160 \mu \mathrm{m}}$. The main northern (IN) and southern (IS) spiral arms are indicated. The angular resolution of $40^{\prime \prime}$ is shown in the lower left corner of the map.

in massive starforming regions, specifically in the southern arm IS and the HII complexes NGC 604, NGC 595, and B690, $\tau_{\mathrm{H} \alpha}$ exceeds 0.7 . The highest dust optical depth one finds in the center of the galaxy and in NGC 604 where $\tau_{\mathrm{H} \alpha} \simeq 0.97$ and 0.88 , respectively (at the linear resolution of $360 \mathrm{pc}$ ). Therefore, M 33 is almost transparent for photons with $\lambda \simeq 6570 \AA$ propagating towards us.

To estimate how much the detected $\mathrm{H} \alpha$ radiation has been attenuated, we note that $\mathrm{H} \alpha$ photons are usually emitted from sources within the galaxy. The optical depth $\tau_{\mathrm{H} \alpha}$ therefore only gives an upper limit. Following Dickinson et al. (2003), we set the effective thickness to $\tau_{\text {eff }}=f_{\mathrm{d}} \times \tau_{\mathrm{H} \alpha}$ with $f_{\mathrm{d}}<1$; the attenuation factor for the $\mathrm{H} \alpha$ flux is then $\mathrm{e}^{-\tau_{\text {eff }}}$.

At $360 \mathrm{pc}$ resolution, one may assume that the $\mathrm{H} \alpha$ emitters, ionized gas in HII regions, and diffuse gas, are uniformly mixed with the dust, which would imply $f_{\mathrm{d}} \simeq 0.5$. Dickinson et al. (2003) find $f_{\mathrm{d}}=0.33$ for the Milky Way (because the 


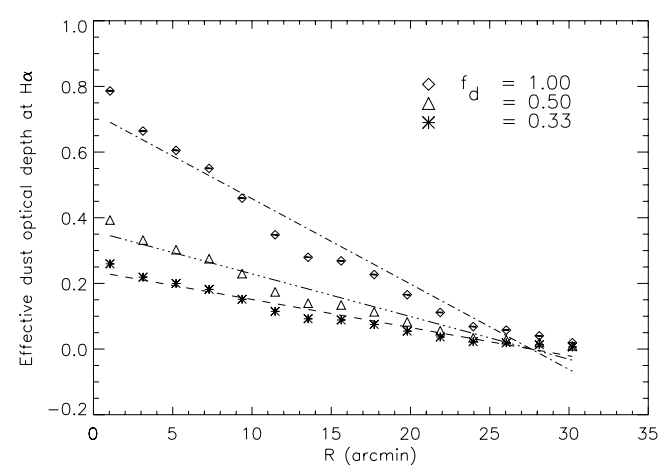

Fig. 4. Radial distribution of the mean effective optical depth at $\mathrm{H} \alpha$ $\left(\tau_{\text {eff }}\right)$ in rings of width of $0.5 \mathrm{kpc}$ in the galactic plane for $f_{\mathrm{d}}=0.33$, 0.5 and 1.0. The errors are smaller than the size of the symbols.

$z$-distribution of the $\mathrm{H} \alpha$ emission is smaller than that of the dust), so we also adopt their value for M33. But, as shown in Sect. 9.4, the determination of the thermal fraction of the radio emission is not very sensitive to the particular choice of $f_{\mathrm{d}}$.

Of course, it would be preferable not to use a uniform value $f_{\mathrm{d}}$ for the whole galaxy, but one that is adapted to the geometry (a well-mixed diffuse medium or shell-like HII regions, Witt \& Gordon 2000) and the dust column density. This needs to specify the location of the stellar sources and the absorbing dust and to solve the radiative transfer problem with massive numerical computations.

An interesting related question is how the extinction in M 33 changes with galactic radius $R$. To investigate this, we integrated Spitzer FIR flux densities at 70 and $160 \mu \mathrm{m}$ in rings of $0.5 \mathrm{kpc}$ width in the galactic plane (inclination of $56^{\circ}$, Regan \& Vogel 1994). We first derived the mean dust temperature of each ring and then the mean dust optical depth $\tau_{\text {eff }}$. Figure 4 shows $\tau_{\text {eff }}$ versus the galactocentric radius $R$. For $f_{\mathrm{d}}=0.33$, the dependence can be described by $\tau_{\mathrm{H}_{\alpha}}(R)=(-0.009 \pm 0.002) R+(0.24 \pm 0.03)$. As $f_{\mathrm{d}}$ rises, the slope gets steeper. The case $f_{\mathrm{d}}=1$ (for the full layer), which is appropriate for pure background sources of M33, is shown for comparison. Generally, a decrease in the extinction with galactocentric radius is expected as it reflects the decrease in surface density towards the periphery.

From a comparison of $\mathrm{H} \alpha$ data with radio flux densities at 6.3 and $21 \mathrm{~cm}$, Israel \& Kennicutt (1980) and Berkhuijsen (1982, 1983 ) found a fairly steep radial gradient of the extinction: considering 8 bright HII regions, Berkhuijsen (1982) derived the relation $\tau_{\mathrm{H} \alpha}=A_{\mathrm{H} \alpha} / 1.086=(-0.038 \pm 0.003) R+(1.45 \pm 0.05)$, with $R$ in arcmin. Qualitatively similar results were reported by Petersen \& Gammelgaard (1997), using Balmer and Paschen emission lines, and by Hippelein et al. (2003) based on a study of the $\mathrm{H} \alpha$ to the $60 \mu \mathrm{m}$ ISOPHOT flux ratio in HII regions. The only discrepant result comes from Devereux et al. (1997) who did not find any systematic decline in $A_{\mathrm{V}}$ from their pixel-topixel comparison of $\mathrm{H} \alpha$ and $6 \mathrm{~cm}$ thermal radio maps. Hippelein et al. (2003) were able to explain it by assuming that the radial gradient of the cold dust $(170 \mu \mathrm{m}$ ISOPHOT) is stronger in the HII regions than in the diffuse gas.

\section{Determination and distribution of free-free emission}

From the observed $\mathrm{H} \alpha$ intensity $I$ and the effective extinction $\tau_{\text {eff }}$, we derived the intrinsic $\mathrm{H} \alpha$ intensity $I_{0}$ according to

$I=I_{0} \mathrm{e}^{-\tau_{\text {eff }}}$.
Integration of the $\mathrm{H} \alpha$ maps out to a radius of $7.5 \mathrm{kpc}$ yields a ratio of corrected-to-observed total $\mathrm{H} \alpha$ flux density of 1.13 for $f_{\mathrm{d}}=0.33$ and 1.25 for $f_{\mathrm{d}}=0.5$. Thus only $13 \%$ (25\%) of the total $\mathrm{H} \alpha$ emission is obscured by dust. The small extinction within M 33 was predicted by the wavelet study of $3.6 \mathrm{~cm}$ and $\mathrm{H} \alpha$ emission (Tabatabaei et al. 2007a).

The emission measure (EM) follows from the $\mathrm{H} \alpha$ intensity via the expression Valls-Gabaud (1998)

$I_{\mathrm{H} \alpha}=9.41 \times 10^{-8} T_{\mathrm{e} 4}^{-1.017} 10^{-\frac{0.029}{T_{\mathrm{e} 4}}} E M$,

where the electron temperature $T_{\mathrm{e} 4}$ is in units of $10^{4} \mathrm{~K}, E M$ in $\mathrm{cm}^{-6} \mathrm{pc}$, and it is assumed that the optical depth of HI resonance lines is large (usually denoted as case B). The emission measure is related to the continuum optical thickness $\tau_{\mathrm{c}}$ of the ionized gas by

$\tau_{\mathrm{c}}=8.235 \times 10^{-2} a T_{\mathrm{e}}^{-1.35} v_{\mathrm{GHz}}^{-2.1}(1+0.08) E M$,

with $a \simeq 1$ (Dickinson et al. 2003). The factor $(1+0.08)$ takes the contribution from the singly ionized He into account. The brightness temperature of the radio continuum emission, $T_{\mathrm{b}}$, then follows from

$T_{\mathrm{b}}=T_{\mathrm{e}}\left(1-\mathrm{e}^{-\tau_{\mathrm{c}}}\right)$.

Whereas the electron temperature in the Milky Way is known to increase with galactocentric radius (Shaver et al. 1983) as a result of decreasing metallicity (Panagia 1979), M 33 does not show any significant variations in metallicity (e.g. Willner \& Nelson-Patel 2002; Magrini et al. 2007). Crockett et al. (2006) have derived the electron temperature from forbidden line ratios in $11 \mathrm{HII}$ regions with galactocentric distances from 1 to $7 \mathrm{kpc}$. Their $T_{\mathrm{e}}$ values range from $7300 \mathrm{~K}$ to $12800 \mathrm{~K}$ with a mean value of $10000 \mathrm{~K}$ and no clear radial gradient. As there are no $T_{\mathrm{e}}$ measurements for the diffuse ionized gas in $\mathrm{M} 33$, we adopt a fixed value of $T_{\mathrm{e}}=10000 \mathrm{~K}$.

The conversion factors from brightness temperature $(\mathrm{K})$ to the observed radio flux density (Jy/beam) are 2.6 and 74.5 at 3.6 and $20 \mathrm{~cm}$, respectively. The final free-free maps are shown in Figs. 5 and 6 . At both wavelengths, the strongest thermal emission emerges from HII regions, in particular, the HII complexes NGC 604 and NGC 595, but the southern arm IS (Paper I) and the center of the galaxy are also very bright. At both wavelengths, the thermal fraction of the diffuse emission is $<25 \%$ in interarm regions. The average error in the thermal fraction obtained using the error propagation method is $7 \%$.

Integrating the thermal maps in rings around the galaxy center out to a radius of $7.5 \mathrm{kpc}$, we obtain the total thermal flux densities and thermal fractions at 3.6 and $20 \mathrm{~cm}$ (see Tables 2 and 3). These tables also give the thermal flux densities and thermal fractions at the position of the 11 brightest HII regions (the coordinates of these HII complexes are listed in Paper I).

\section{Distribution of nonthermal emission}

Maps of the nonthermal emission are obtained by subtracting the maps of the thermal emission from the observed maps at each wavelength (Figs. 5 and 6). The nonthermal maps exhibit diffuse emission extending to large radii. They also show strong features in the spiral arms and central region of the galaxy. The strongest nonthermal emission emerges from the HII complexes NGC 604 and NGC 595 at both 3.6 and $20 \mathrm{~cm}$. Typically, HII complexes host tens of young O/B stars, many of which end as supernovae whose remnants contribute to a mixed (thermal and nonthermal), 

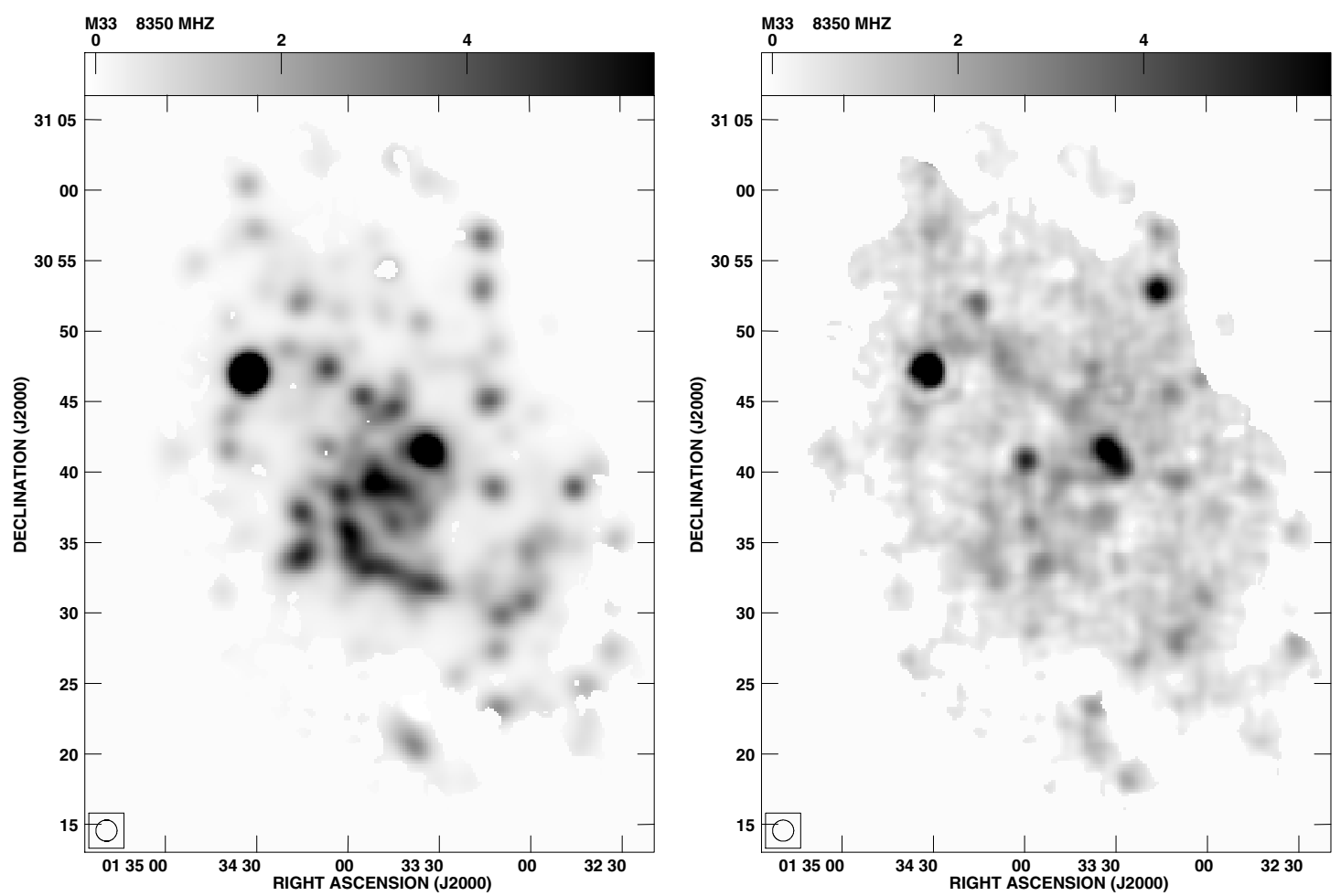

Fig. 5. Thermal (left) and nonthermal (right) maps at $3.6 \mathrm{~cm}$. The grey scale gives the flux density in mJy/beam. The angular resolution is $90^{\prime \prime}$ (shown in the lower left of the images) with a grid size of $10^{\prime \prime}$. The bright HII complexes NGC $604\left(\mathrm{RA}=1^{\mathrm{h}} 34^{\mathrm{m}} 32.9^{\mathrm{s}}\right.$ and Dec $\left.=30^{\circ} 47^{\prime} 19.6^{\prime \prime}\right)$ and NGC $595\left(\mathrm{RA}=1^{\mathrm{h}} 33^{\mathrm{m}} 32.4^{\mathrm{s}}\right.$ and $\left.\mathrm{Dec}=30^{\circ} 41^{\prime} 50.0^{\prime \prime}\right)$ are clearly visible in both maps, whereas IC $133\left(\mathrm{RA}=1^{\mathrm{h}} 33^{\mathrm{m}} 15.3^{\mathrm{s}}\right.$ and $\left.\mathrm{Dec}=30^{\circ} 53^{\prime} 19.7^{\prime \prime}\right)$ appears stronger in the nonthermal map.
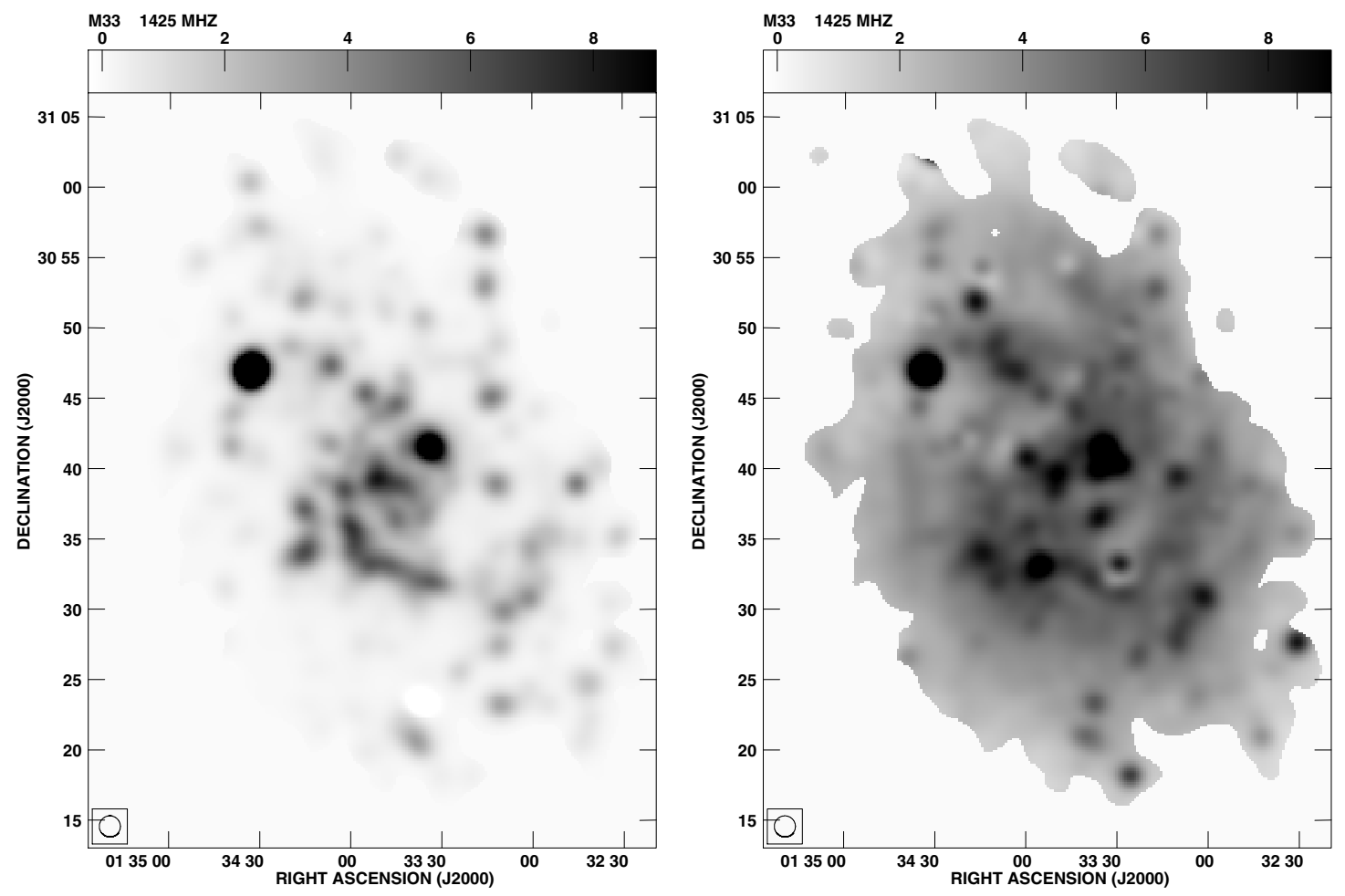

Fig. 6. Thermal (left) and nonthermal (right) maps at $20 \mathrm{~cm}$. The grey scale gives the flux density in mJy/beam. The angular resolution is $90^{\prime \prime}$ (shown in the lower left of the images) with a grid size of $10^{\prime \prime}$. 
Table 2. Thermal flux density and thermal fraction at $3.6 \mathrm{~cm}\left(f_{\mathrm{d}}=0.33\right.$ and $\left.T_{\mathrm{e}}=10000 \mathrm{~K}\right)$.

\begin{tabular}{cccc}
\hline \hline Object & $\begin{array}{c}\text { Observed } \\
\text { flux density } \\
\text { (mJy) }\end{array}$ & $\begin{array}{c}\text { Thermal } \\
\text { flux density } \\
\text { (mJy) }\end{array}$ & $\begin{array}{c}\text { Thermal } \\
\text { fraction } \\
\%\end{array}$ \\
\hline NGC 604 & $50.44 \pm 0.13$ & $38.55 \pm 0.08$ & $76.4 \pm 0.3$ \\
NGC 595 & $17.95 \pm 0.18$ & $12.13 \pm 0.11$ & $67.6 \pm 1.3$ \\
IC 133 & $9.08 \pm 0.11$ & $2.66 \pm 0.04$ & $29.3 \pm 0.8$ \\
B690 & $4.51 \pm 0.11$ & $3.11 \pm 0.10$ & $68.9 \pm 3.9$ \\
B61/62 & $3.72 \pm 0.11$ & $2.24 \pm 0.07$ & $60.2 \pm 3.7$ \\
IC 132 & $4.89 \pm 0.05$ & $3.25 \pm 0.02$ & $66.5 \pm 1.0$ \\
IC 131 & $4.59 \pm 0.07$ & $3.26 \pm 0.05$ & $71.0 \pm 2.2$ \\
NGC 588 & $4.64 \pm 0.09$ & $3.24 \pm 0.06$ & $69.8 \pm 2.6$ \\
IC 142 & $3.51 \pm 0.08$ & $2.73 \pm 0.10$ & $77.8 \pm 4.6$ \\
B691 & $4.60 \pm 0.13$ & $2.35 \pm 0.06$ & $51.1 \pm 2.6$ \\
NGC 592 & $4.28 \pm 0.08$ & $2.76 \pm 0.06$ & $64.5 \pm 2.6$ \\
\hline M 33 (mJy): & & & \\
$R<7.5 \mathrm{kpc}$ & $761 \pm 63$ & $391 \pm 74$ & $51.4 \pm 4.2$ \\
\hline
\end{tabular}

Table 3. Thermal flux density and thermal fraction at $20 \mathrm{~cm}\left(f_{\mathrm{d}}=0.33\right.$ and $T_{\mathrm{e}}=10000 \mathrm{~K}$ ).

\begin{tabular}{cccc}
\hline \hline Object & $\begin{array}{c}\text { Observed } \\
\text { flux density } \\
\text { (mJy) }\end{array}$ & $\begin{array}{c}\text { Thermal } \\
\text { flux density } \\
\text { (mJy) }\end{array}$ & $\begin{array}{c}\text { Thermal } \\
\text { fraction } \\
\%\end{array}$ \\
\hline NGC 604 & $62.75 \pm 0.21$ & $45.46 \pm 0.09$ & $72.4 \pm 0.4$ \\
NGC 595 & $20.50 \pm 0.22$ & $14.31 \pm 0.14$ & $69.8 \pm 1.4$ \\
IC133 & $5.35 \pm 0.07$ & $3.11 \pm 0.03$ & $58.1 \pm 0.9$ \\
B690 & $5.80 \pm 0.12$ & $3.69 \pm 0.12$ & $63.6 \pm 2.4$ \\
B61/62 & $4.00 \pm 0.21$ & $2.69 \pm 0.08$ & $67.2 \pm 4.0$ \\
IC 132 & $5.52 \pm 0.05$ & $3.81 \pm 0.02$ & $69.0 \pm 0.7$ \\
IC 131 & $4.12 \pm 0.07$ & $3.84 \pm 0.06$ & $93.2 \pm 2.1$ \\
NGC 588 & $4.67 \pm 0.17$ & $3.74 \pm 0.06$ & $80.0 \pm 3.2$ \\
IC142 & $4.14 \pm 0.08$ & $3.09 \pm 0.13$ & $74.6 \pm 3.4$ \\
B691 & $7.40 \pm 0.20$ & $2.43 \pm 0.04$ & $32.8 \pm 1.0$ \\
NGC 592 & $5.43 \pm 0.14$ & $3.27 \pm 0.08$ & $60.2 \pm 2.1$ \\
\hline M33 (mJy): & & & \\
$R<7.5 \mathrm{kpc}$ & $2722 \pm 60$ & $478 \pm 85$ & $17.6 \pm 0.4$ \\
\hline
\end{tabular}

flat spectrum of the total radio emission from these regions (as discovered in NGC 604 and NGC 595, Dodorico 1978; Gordon et al. 1993; Yang et al. 1996). Supernova remnants with central energy sources in the form of young pulsars (Crab-like remnants) also have flat nonthermal spectra.

Another strong feature in the $20 \mathrm{~cm}$ nonthermal map belongs to the active starforming region in the central southern arm IS. This confirms our previous conclusion about the spatial coupling of the nonthermal emission with the starforming regions (Paper I). There are also other point-like sources that are not resolved. Looking at the $3.6 \mathrm{~cm}$ map, the giant HII region IC $133\left(\mathrm{RA}=1^{\mathrm{h}} 33^{\mathrm{m}} 15.3^{\mathrm{s}}\right.$ and $\left.\mathrm{Dec}=30^{\circ} 53^{\prime} 19.7^{\prime \prime}\right)$ is also strong, but not at $20 \mathrm{~cm}$. Hosting the two strongest optically thick HII regions (Johnson et al. 2001), IC 133 has an inverted spectrum. The appearance of this source in the $3.6 \mathrm{~cm}$ nonthermal map is due to the assumption that the free-free emission is optically thin (equivalent to the thermal spectral index of 0.1 when $\left.S_{\text {th }} \sim v^{-0.1}\right)$. Furthermore, IC 133 probably contains some nonthermal emission as Schulman \& Bregman (1995) found it associated with a bright X-ray source located in a hole in the HI layer of the galaxy, indicating energetic stellar winds and supernovae from massive stars.

Tabatabaei et al. (2007a) discussed the wavelet spectrum of the total radio continuum maps, while here we present the wavelet energy of the thermal and nonthermal emission on

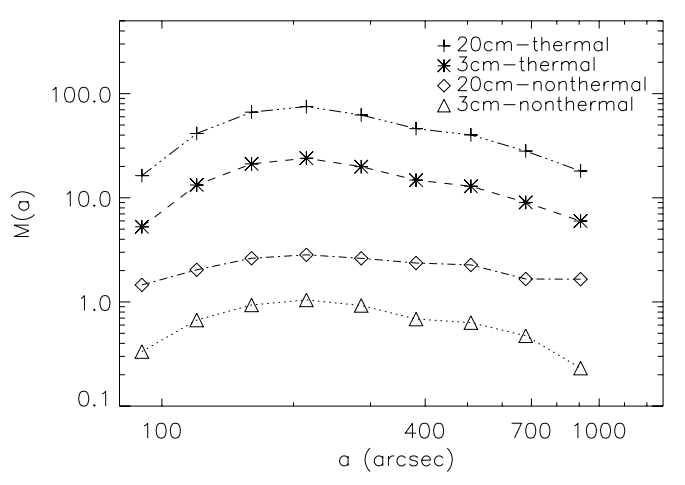

Fig. 7. The wavelet spectra of the 3.6 and $20 \mathrm{~cm}$ thermal and nonthermal emission at $90^{\prime \prime}$ resolution. The data points correspond to the scales $90,120,160,214,286,381,509,679,907^{\prime \prime}$. The spectra are shown in arbitrary units, and $100^{\prime \prime}$ corresponds to $400 \mathrm{pc}$ in M 33 .

different scales. Figure 7 shows that the wavelet spectrum of the nonthermal emission is smoother than for the thermal emission at both wavelengths. Furthermore, the distribution of the nonthermal wavelet energy is smoother at $20 \mathrm{~cm}$ than at $3.6 \mathrm{~cm}$. Scales smaller than the width of the spiral arms $\left(\simeq 400^{\prime \prime}\right.$ or $1.6 \mathrm{kpc})$ and larger than the giant starforming regions $\left(\simeq 100^{\prime \prime}\right.$ or $0.4 \mathrm{kpc}$ ) at $20 \mathrm{~cm}$ are not as prominent as those at $3.6 \mathrm{~cm}$, where the synchrotron photons have higher energies. In fact the synchrotron emission distribution reflects the combined distribution of the interstellar magnetic field and the cosmic ray electrons. Hence, assuming that the distribution of the interstellar magnetic field is the same at both wavelengths, one can conclude: the higher the energy of CRs, the more dominant the localized structures (which can be linked to the cosmic ray "sources" e.g. supernova remnants). This is expected, as energy losses of CR electrons increase with electron energy and field strength. Studying propagation effects with self-consistent Galactic wind simulations, Breitschwerdt et al. (2002) predicted a similar energy dependence for the CR nucleon distribution in our Galaxy. They expect that the high-energy $(\mathrm{TeV}) \gamma$-rays from CR sources dominate the diffuse $\gamma$-ray emission, while the Galactic $\gamma$-ray observations in the GeV range (with EGRET and COS-B) have shown a roughly uniform distribution of the $\gamma$-ray emissivity in the Galactic plane.

The dominant scales of the wavelet spectra of the thermal (at 3.6 and $20 \mathrm{~cm}$ ) and the $3.6 \mathrm{~cm}$ nonthermal emission are the same, indicating that the distribution of the cosmic ray sources is similar to that of the thermal sources. On scales larger than $a=214^{\prime \prime}$, the $3.6 \mathrm{~cm}$ nonthermal spectrum decreases twice as fast as the $20 \mathrm{~cm}$ nonthermal spectrum. The smaller energy losses of the CR electrons with lower energies (at $20 \mathrm{~cm}$ ) than of those with higher energies (at $3.6 \mathrm{~cm}$ ) may cause a smoother distribution of the emission on all scales. This indicates that, besides the CR sources embedded in the starforming regions, there is another component of the synchrotron emission in the form of a diffuse disk or a galactic halo that is more visible at $20 \mathrm{~cm}$ than $3.6 \mathrm{~cm}$. This could be verified by multiwavelength observations of edge-on galaxies at resolutions higher than those of the existing data.

Figure 8 shows the the supernova remnants from Gordon et al. (1998) superimposed on the nonthermal $20 \mathrm{~cm}$ map. There is general coincidence between nonthermal features and supernova remnants, the powerful sources of the relativistic electrons. Gordon et al. (1999) diagnosed 17 supernova remnants 


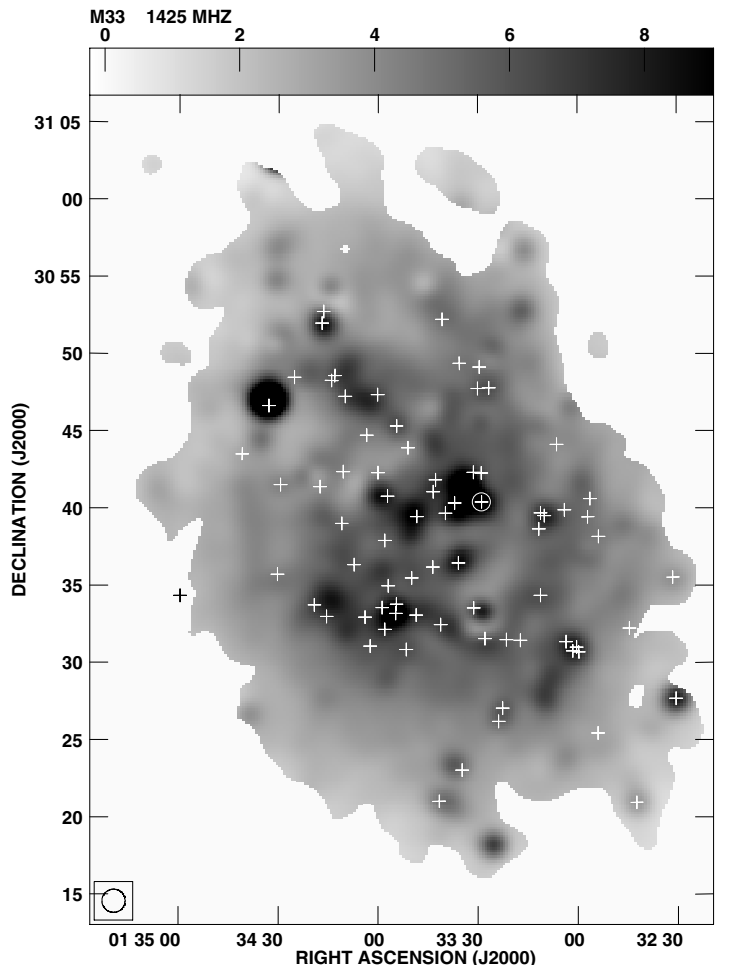

Fig. 8. The same $20 \mathrm{~cm}$ nonthermal map as shown in Fig. 6 with supernova remnants (crosses) superimposed. The supernova remnants with a signal-to-noise ratio higher than 0.7 were selected from the catalogue of Gordon et al. (1998). The circle shows an HII region with nonthermal spectral index (Gordon et al. 1999).

embedded in HII regions. They also found some 30 HII regions with nonthermal radio components where it was not clear whether they belong to these regions or were external radio sources.

\section{Nonthermal spectral index}

From the nonthermal radio fluxes at 3.6 and $20 \mathrm{~cm}$, we obtained the spectral index of the nonthermal emission, which was only computed for pixels with flux densities of at least three times the rms noise $\sigma$ at both frequencies. Figure 9 shows that the nonthermal spectral index $\alpha_{n}$ has a clumpy distribution. Note that the fainter regions have steeper spectra. The most probable value of $\alpha_{n}$ distributed across the galaxy is 0.95 (see the lower panel in Fig. 9). In the starforming regions, the nonthermal spectrum is relatively flat with an average value of $\alpha_{n}$ of $0.6 \pm 0.1$, the typical spectral index of supernova remnants, but $\alpha_{n}$ increases to $1.2 \pm 0.2$ in the interarm regions and outer parts of the galaxy. This indicates energy losses of the relativistic electrons while they diffuse away from their origin in starforming regions towards the interarm regions and the outer parts of the galaxy. For the first time, a nonthermal spectral index map can be used to achieve more realistic models for the propagation of CR electrons.

Integration of the nonthermal intensity in rings of $0.5 \mathrm{kpc}$ in the galactic plane yields the mean nonthermal spectral index in each ring. The radial variation of the ring mean spectral index is shown in Fig. 10, where the ring mean spectral index of the total radio continuum emission is also plotted for comparison. Up to $R \simeq 4 \mathrm{kpc}$, there is no increasing trend for the nonthermal spectral index with radius as for the total spectral index. Here the ring
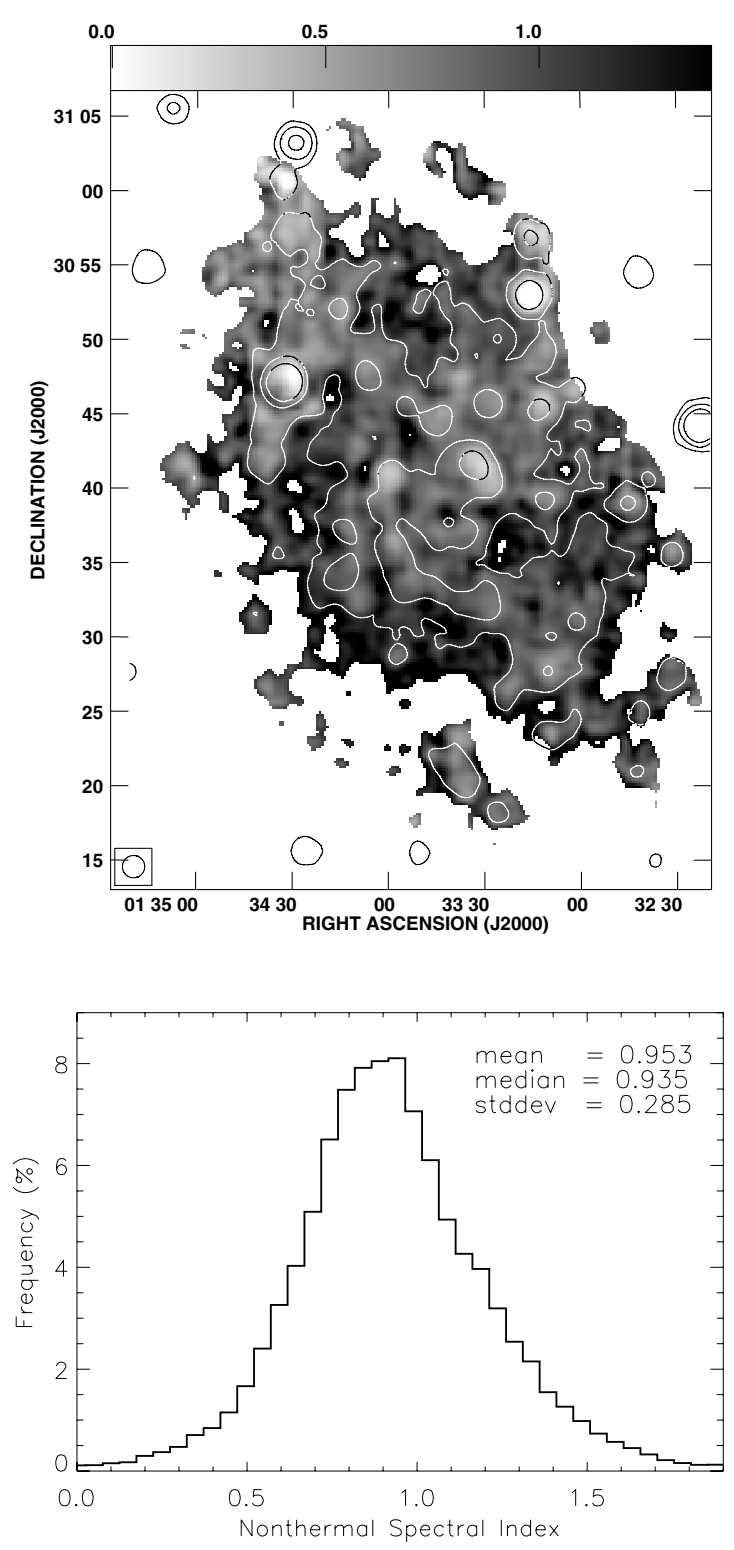

Fig. 9. Nonthermal spectral index $\left(\alpha_{n}\right)$ map obtained from the nonthermal radio fluxes at 3.6 and $20 \mathrm{~cm}$. The spiral arms are indicated by contours of the total radio emission at $3.6 \mathrm{~cm}$ superimposed. Contour levels are $1.5,4.5$, and $12 \mathrm{mJy} / \mathrm{beam}$. The angular resolution is $90^{\prime \prime}$ with a grid size of $10^{\prime \prime}$. Bottom: a histogram of the nonthermal spectral index map. The number of bins used for this plot is 50 .

mean nonthermal spectral index varies between 0.65 and 0.9 , indicating that CRs are injected by sources related to massive stars and under leakage loss (Biermann 1995). Towards the outer parts of the galaxy, the nonthermal and total spectral indices converge, confirming that the total radio continuum emission is mostly nonthermal at $R>4.5 \mathrm{kpc}$. This region corresponds to the synchrotron- (and inverse Compton-) loss-dominated regime as $\alpha_{n} \simeq 1$ on average (Biermann 1995).

\section{Discussion}

\subsection{Comparison with the standard method}

In this section, we first obtain the distribution of the thermal and nonthermal emission by assuming that the nonthermal spectral 


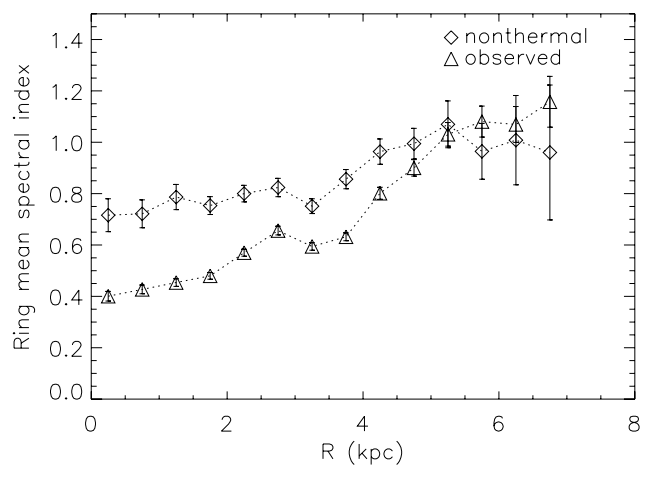

Fig. 10. Mean nonthermal spectral index in rings of $0.5 \mathrm{kpc}$ width in the galactic plane versus galactocentric radius. The total (observed) spectral index is also shown for comparison. The minima at $3<R<4 \mathrm{kpc}$ is due to the HII complex NGC 604.

index is constant across the galaxy (standard method). Then we compare the results from both methods.

Because the thermal emission is weak in the outer parts of the galaxy, one may consider the total spectral index from these parts as the pure nonthermal spectral index ${ }^{4}, \alpha_{n}$ (e.g. Berkhuijsen et al. 2003). The total spectral index map of M 33 gives $\alpha_{n}=1.0 \pm 0.1$ (see Fig. 11, Tabatabaei et al. 2007b). For a total spectral index $\alpha$ obtained from the observed flux densities at frequencies $v_{1}$ and $v_{2}$ and the constant value of $\alpha_{n}$, the thermal fraction at frequency $v_{1}$ is given by

$F_{\mathrm{th}}^{v_{1}}=\left(\left(\frac{v_{2}}{v_{1}}\right)^{-\alpha}-\left(\frac{v_{2}}{v_{1}}\right)^{-\alpha_{n}}\right) /\left(\left(\frac{v_{2}}{v_{1}}\right)^{-0.1}-\left(\frac{v_{2}}{v_{1}}\right)^{-\alpha_{n}}\right)$

(Klein et al. 1984). Then the thermal flux density at frequency $v_{1}$, $S_{\text {th }}^{v_{1}}$, is obtained from

$S_{\text {th }}^{v_{1}}=S^{v_{1}} \times F_{\text {th }}^{\nu_{1}}$,

and the nonthermal flux density at frequency $v_{1}, S_{n}^{\nu_{1}}$ is

$S_{n}^{\nu_{1}}=S^{\nu_{1}}-S_{\mathrm{th}}^{\nu_{1}}$

Using the data at 3.6 and $20 \mathrm{~cm}$ in the above formulae, the corresponding thermal and nonthermal maps are derived; those at $20 \mathrm{~cm}$ are shown in Fig. 11. The main difference between the standard method and our new one (Fig. 6) concerns the distribution of the nonthermal emission, while the thermal maps show almost the same structures. The nonthermal emission from the standard method is weaker than the one from the new method and hardly shows emission from starforming regions in the arms. Often it is even weaker in the arms than between the arms and in the outer parts of the galaxy.

In contrast to what is assumed in the standard method, large variations in the nonthermal spectral index are found across M33 by the new method (Fig. 9). We interpret this as clear evidence that $\mathrm{CR}$ electrons suffer energy losses diffusing away from their places of origin in the arms towards interarm and outer regions.

Table 4 lists the thermal fractions of the 11 bright HII regions and of M 33 (from the integrated flux density maps up to $R=7.5 \mathrm{kpc}$ ) at $3.6 \mathrm{~cm}$ obtained from both methods. The thermal fractions of all the sources are larger when obtained by the standard method than by the new method. Some even exceed $100 \%$,

\footnotetext{
4 This is confirmed by the new method, as mentioned in Sect. 8.
}

causing very weak or negative nonthermal fluxes. The thermal fraction of M 33 by the standard method is $23 \% \pm 14 \%$ higher than from the new method. In Paper I, we used the standard method with $\alpha_{n}=1.0 \pm 0.1$ to estimate the thermal fraction from the integrated spectrum, based on data at 35.6, 21.1, 17.4, $11.1,6.3,6.2,3.6$, and $2.8 \mathrm{~cm}$, as $0.49 \pm 0.15$, which agrees with the value $0.51 \pm 0.04$ obtained from the new method. This indicates that the assumption of a constant nonthermal spectral index is reasonable for estimating the mean values for global studies, when the integrated spectrum is used.

\subsection{Radial scale lengths}

Figure 12 shows the exponential distributions of the thermal and nonthermal intensities at 3.6 and $20 \mathrm{~cm}$ with galactocentric radius. The radial profiles of the nonthermal emission are smoother and flatter than those of the thermal emission at both wavelengths. The fluctuations in the thermal profiles at $2<R<4 \mathrm{kpc}$ cause similar fluctuations observed in the profile of the total emission (presented in Tabatabaei et al. 2007b).

The same profiles obtained from the standard method are shown in Fig. 13 for comparison. The nonthermal radial profiles are slightly smoother (especially at $3.6 \mathrm{~cm}$ ) than those of the new method, caused by the assumption of a constant nonthermal spectral index. Although the thermal radial profiles exhibit the same variations as those in Fig. 12, the overestimation of the thermal emission from the standard method is obvious.

The exponential scale length $l$ is obtained by fitting an exponential function of the form $I(\mathrm{R})=I_{0} \exp (-R / l)$, where $I_{0}$ is the intensity at $R=0$. Table 5 shows the exponential scale lengths of the thermal emission, $l_{\mathrm{th}}$, and nonthermal emission, $l_{n}$, from both methods. Generally, the scale lengths of the nonthermal emission are larger than those of the thermal emission (by a factor of $\simeq 2$ ). In Sect. 7, we show that the CR sources follow the distribution of the thermal sources, i.e. starforming regions. Hence, the radial distribution of the $\mathrm{CR}$ sources decreases faster than that of the synchrotron emission. This is a direct observational result indicating diffusion of the cosmic rays from their places of origin to greater distances.

Using the standard method, Buczilowski (1988) determined the scale length of the $6.3 \mathrm{~cm}$ thermal and nonthermal emission as $1.8 \pm 0.2 \mathrm{kpc}$ and $4.2 \pm 0.3 \mathrm{kpc}$, respectively. These scale lengths are smaller than those obtained here (even smaller than those obtained from the standard method), although the ratio of the nonthermal to thermal scale lengths is the same $(\simeq 2)$. Due to the low signal-to-noise ratio of the old $6.3 \mathrm{~cm}$ Effelsberg receiver used by Buczilowski (1988), much of the diffuse emission in the outer parts of the galaxy was missed, leading to steeper radial profiles and smaller scale lengths. For the same reason, the scale lengths derived by Berkhuijsen \& Klein (1985) are also too small. They obtained the distribution of the thermal emission at $6.2 \mathrm{~cm}$ from a catalogue of HII regions in $\mathrm{H} \alpha$ (Boulesteix et al. 1974) where the diffuse emission was not completely included.

In case of equipartition between the magnetic field and CRs, the scale length of the CR electrons is given by $l_{\mathrm{cr}}=l_{n}\left(3+\alpha_{n}\right) / 2$ and that of the magnetic field by $l_{\mathrm{B}}=2 l_{\mathrm{cr}}$ (e.g. Klein et al. 1982). Taking $\alpha_{n} \simeq 1$, we obtain $l_{\text {cr }} \simeq 12 \mathrm{kpc}$ and $l_{\mathrm{B}} \simeq 24 \mathrm{kpc}$.

For NGC 6946, Walsh et al. (2002) find a nonthermal scale length of $l_{n} \sim 4 \mathrm{kpc}$, which gives a smaller CR scale length of $l_{\text {cr }} \simeq 8 \mathrm{kpc}$. Although NGC 6946 is a Scd-type galaxy like M 33, it is a starburst system and it hosts stronger starformation in its central region than M33. This may cause a steeper radial profile of the total intensity and consequently a smaller nonthermal (and cosmic ray) scale length than in M 33 . 

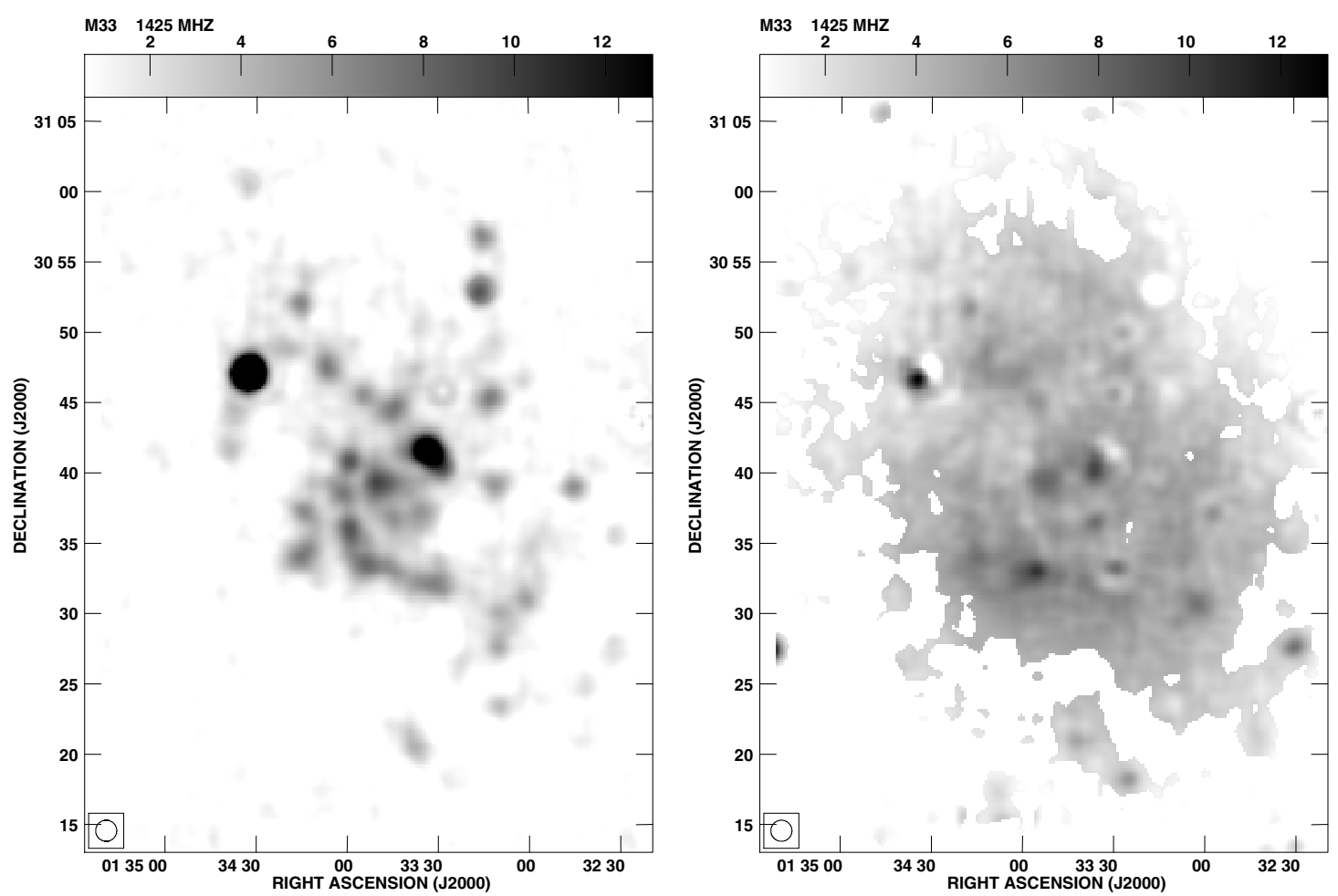

Fig. 11. Thermal and nonthermal $20 \mathrm{~cm}$ maps obtained from the standard method. The grey-scale gives the flux density in mJy/beam. The angular resolution is $90^{\prime \prime}$ with a grid size of $10^{\prime \prime}$.
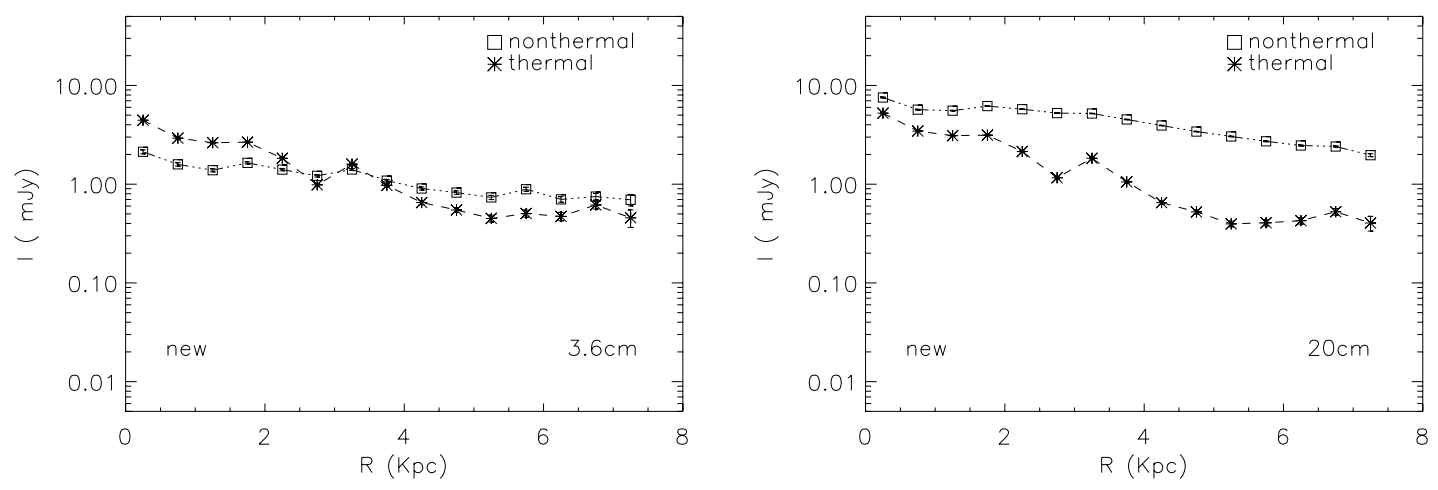

Fig. 12. Radial profiles of the flux densities of the thermal and nonthermal emission at $3 \mathrm{~cm}(l e f t)$ and $20 \mathrm{~cm}$ (right) obtained from the new separation method. The thermal flux densities show a bump at $3<R<4 \mathrm{kpc}$, which is due to the HII complex NGC 604 .
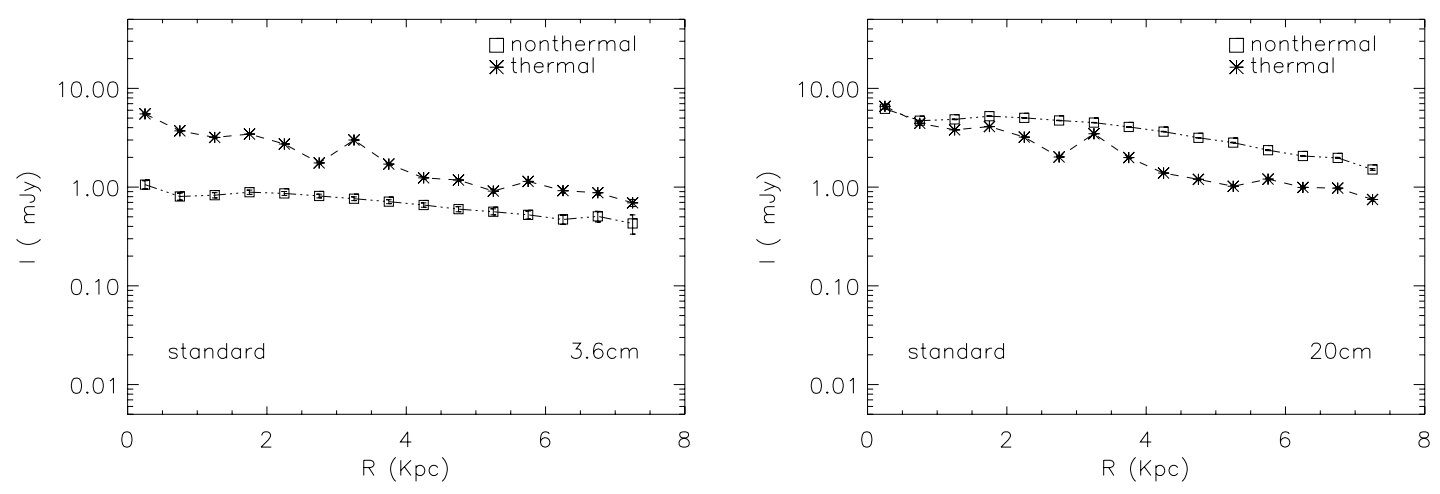

Fig. 13. Radial profiles of the flux densities of the thermal and nonthermal emission at $3 \mathrm{~cm}(l e f t)$ and $20 \mathrm{~cm}$ (right) obtained from the standard separation method. The thermal flux densities show a bump at $3<R<4 \mathrm{kpc}$ that is due to the HII complex NGC 604 . 
Table 4. Thermal fractions at $3.6 \mathrm{~cm}$ obtained from the new and standard methods.

\begin{tabular}{cccc}
\hline \hline Object & $\alpha_{(3.6,20)}$ & $\begin{array}{c}F_{\text {th }}(\text { new } \\
\text { method) } \%\end{array}$ & $\begin{array}{c}F_{\text {th }} \text { (standard } \\
\text { method) } \%\end{array}$ \\
\hline NGC 604 & $0.12 \pm 0.01$ & $76.4 \pm 0.3$ & $99.2 \pm 0.4$ \\
NGC 595 & $0.07 \pm 0.03$ & $67.6 \pm 1.3$ & $101.6 \pm 1.4$ \\
IC133 & $-0.30 \pm 0.03$ & $29.3 \pm 0.8$ & $106.5 \pm 1.6$ \\
B690 & $0.14 \pm 0.05$ & $68.9 \pm 3.9$ & $99.1 \pm 3.6$ \\
B61/62 & $0.04 \pm 0.08$ & $60.2 \pm 3.7$ & $104.3 \pm 4.2$ \\
IC 132 & $0.07 \pm 0.02$ & $66.5 \pm 1.0$ & $101.6 \pm 1.5$ \\
IC 131 & $-0.06 \pm 0.03$ & $71.0 \pm 2.2$ & $107.2 \pm 2.4$ \\
NGC 588 & $0.00 \pm 0.05$ & $69.8 \pm 2.6$ & $103.1 \pm 2.9$ \\
IC 142 & $0.09 \pm 0.04$ & $77.8 \pm 4.6$ & $96.9 \pm 3.6$ \\
B691 & $0.27 \pm 0.05$ & $51.1 \pm 2.6$ & $93.0 \pm 3.4$ \\
NGC 592 & $0.13 \pm 0.04$ & $64.5 \pm 2.6$ & $97.9 \pm 2.8$ \\
\hline M33: & & & \\
$R<7.5 \mathrm{kpc}$ & $0.72 \pm 0.04$ & $51.4 \pm 4.2$ & $63.2 \pm 5.3$ \\
\hline
\end{tabular}

Table 5. Exponential scale lengths of the thermal and nonthermal components of the radio contiuum emission from M 33 .

\begin{tabular}{ccc}
\hline \hline$\lambda$ & $l_{\text {th }}(\mathrm{kpc})$ & $l_{n}(\mathrm{kpc})$ \\
\hline new method & & \\
$20 \mathrm{~cm}$ & $2.4 \pm 0.2$ & $5.8 \pm 0.5$ \\
$3.6 \mathrm{~cm}$ & $2.6 \pm 0.2$ & $6.1 \pm 0.7$ \\
\hline standard method & & \\
$20 \mathrm{~cm}$ & $3.5 \pm 0.5$ & $6.2 \pm 0.7$ \\
$3.6 \mathrm{~cm}$ & $3.7 \pm 0.5$ & $8.9 \pm 0.9$ \\
\hline
\end{tabular}

\subsection{North-south asymmetry}

From Figs. 5 and 6 it seems that the thermal emission is stronger in the southern than in the northern half of M33. To investigate this north-south (N-S) asymmetry, we obtained the integrated flux density of the thermal, nonthermal, and total emission in each half separately. Table 6 shows the results from both the new and the standard methods. The thermal emission from the new method is slightly stronger in the southern than in the northern half of the galaxy at both wavelengths. This may be the reason for the higher Faraday depolarization found in the southern half than in the northern half (Tabatabaei et al. 2007b). In Paper III, we will discuss whether the asymmetry in the Faraday depolarization can be caused by this $\mathrm{N}-\mathrm{S}$ asymmetry in the thermal emission distribution. Note that, by the standard method, the thermal emission in the southern half is weaker.

The nonthermal emission at $20 \mathrm{~cm}$ from the new method in the southern half is also stronger than in the northern half (in contrast to the $3.6 \mathrm{~cm}$ nonthermal emission). This is expected as there are more supernova remnants in the southern half, $\simeq 56 \%$ of the total number, especially in the main southern arm IS (see Fig. 8), which produce stronger nonthermal emission at longer wavelengths. The existence of the strong optically thick HII regions (e.g. IC 133) in the northern half of the galaxy also causes the different $\mathrm{N}-\mathrm{S}$ ratios of the nonthermal emission at $3.6 \mathrm{~cm}$.

\subsection{Uncertainties}

How do the assumptions of the new method of separating thermal and nonthermal emission influence the results? We take the thermal fraction (e.g. at $3.6 \mathrm{~cm}$ ) as the final result and define $U$ as the uncertainty in $F_{\text {th }}$ when one of the assumptions changes,

$U \equiv \frac{\left|F_{\mathrm{th}}-F_{\mathrm{th}}^{\prime}\right|}{F_{\mathrm{th}}}$
Table 6. North-South ratios of the integrated flux densities of the thermal, nonthermal, and total radio emission.

\begin{tabular}{cccc}
\hline \hline$\lambda(\mathrm{cm})$ & $S_{\mathrm{th}}^{\mathrm{N}} / S_{\mathrm{th}}^{\mathrm{S}}$ & $S_{n}^{\mathrm{N}} / S_{n}^{\mathrm{S}}$ & $S_{\mathrm{T}}^{\mathrm{N}} / S_{\mathrm{T}}^{\mathrm{S}}$ \\
\hline new method & & & \\
3.6 & $0.89 \pm 0.05$ & $1.15 \pm 0.08$ & $1.10 \pm 0.08$ \\
20 & $0.86 \pm 0.05$ & $0.75 \pm 0.07$ & $0.82 \pm 0.07$ \\
\hline standard method & & & \\
3.6 & $1.20 \pm 0.07$ & $0.67 \pm 0.07$ & $1.10 \pm 0.08$ \\
20 & $1.14 \pm 0.06$ & $0.77 \pm 0.08$ & $0.82 \pm 0.07$ \\
\hline
\end{tabular}

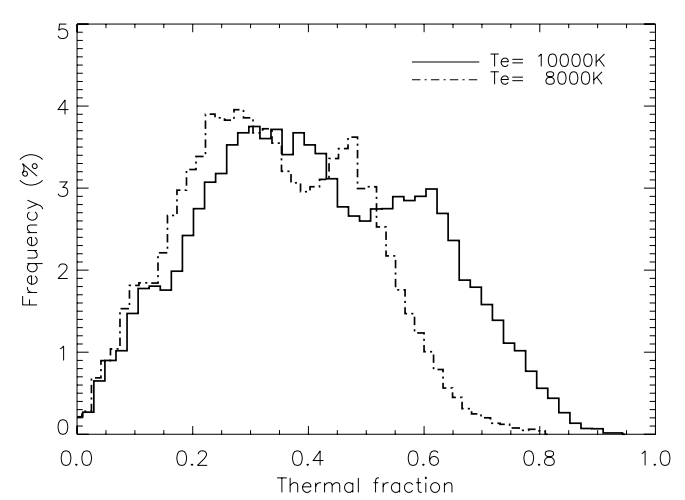

Fig. 14. Distribution (histogram) of the thermal fraction at $3.6 \mathrm{~cm}$ in M33. The number of bins used for this histogram is 50 .

where $F_{\text {th }}$ and $F_{\text {th }}^{\prime}$ are the thermal fractions before and after changing an assumption, respectively. One of these assumptions is the choice of effective extinction factor $f_{\mathrm{d}}=0.33$ (non-uniform ionization). For a homogeneous distribution of dust and ionized gas, $f_{\mathrm{d}}=0.5$, the average uncertainty in the thermal fraction at $3.6 \mathrm{~cm}$ is only $U=2 \%$ with a standard deviation of $1 \%$.

Changing $T_{\mathrm{e}}$ from the adopted $10000 \mathrm{~K}$ to $8000 \mathrm{~K}$, the most probable uncertainty across the galaxy is $U=17 \%$ with a standard deviation of $3 \%$. Figure 14 shows that the thermal fraction decreases with decreasing electron temperature. Larger differences in the thermal fraction are found for regions with higher thermal emission like HII regions, and the influence of chang$\operatorname{ing} T_{\mathrm{e}}$ is relatively weak in diffuse regions with small $F_{\text {th }}$. Thus, we find that the uncertainty resulting from the new method is mainly determined by the uncertainty of the electron temperature.

Another question is which method faces larger uncertainty in the thermal fraction, the new method with its electron temperature assumption or the standard method with its constant nonthermal spectral index assumption. For this comparison, we first obtained the nonthermal spectral indices for different electron temperatures. For $T_{\mathrm{e}}=10000 \mathrm{~K}$ the most probable nonthermal spectral index is 0.9 , while it is 0.8 for $T_{\mathrm{e}}=8000 \mathrm{~K}$. Then, from the standard method, we calculated the thermal fractions assuming $\alpha_{n}=0.9$ and 0.8 , respectively, which leads to an uncertainty of $U=40 \%$, much larger than what determined with our method $(17 \%)$. We conclude that the thermal fraction is more sensitive to variations in the nonthermal spectral index in the standard method than to variations in the electron temperature in the new method.

Table 7 shows the thermal fractions obtained from the thermal and total flux densities of M 33 (integrated for $R<7.5 \mathrm{kpc}$ ) for different combinations of the assumptions of $T_{\mathrm{e}}$ and $f_{\mathrm{d}}$. The uncertainty $U$ of each assumption is calculated with respect to the case $T_{\mathrm{e}}=10000 \mathrm{~K}$ and $f_{\mathrm{d}}=0.33$. 
Table 7. Average thermal fraction of M33 at $3.6 \mathrm{~cm}$ for different assumptions.

\begin{tabular}{cccc}
\hline \hline$T_{\mathrm{e}}$ & $f_{\mathrm{d}}$ & $F_{\text {th }}$ & $U$ \\
$(\mathrm{~K})$ & $(\ldots)$ & $(\%)$ & $(\%)$ \\
\hline 10000 & 0.33 & 51 & $(\ldots)$ \\
10000 & 0.50 & 54 & 5 \\
8000 & 0.33 & 41 & 20 \\
8000 & 0.50 & 43 & 16 \\
\hline
\end{tabular}

\section{Summary and conclusions}

We have developed a new method of separating the thermal and nonthermal radio emission from a galaxy. We used the highly resolved and sensitive Spitzer 70 and $160 \mu \mathrm{m}$ data of M 33 to correct the $\mathrm{H} \alpha$ map of Hoopes \& Walterbos (2000) for extinction. From this map, we calculated the thermal (free-free) emission at 3.6 and $20 \mathrm{~cm}$ and obtained maps of the nonthermal emission, as well as a map of the nonthermal spectral index in M33. The distribution of the nonthermal spectral index greatly helps in understanding the origin and propagation of cosmic ray electrons in a galaxy. In brief, the results and conclusions are as follows.

- The distribution of the dust extinction is similar to that of the $160 \mu \mathrm{m}$ emission. The mean extinction in rings in the galactic plane exhibits a shallow radial gradiant.

- With a nonthermal fraction of about $30 \%-60 \%$ at $3.6 \mathrm{~cm}$, the spiral arms and starforming regions make a considerable contribution to the nonthermal emission. This contribution is negligible in the nonthermal maps obtained from the standard separation method. The radial profiles of the surface brightnesses and the wavelet spectra show that the distribution of the nonthermal emission from the standard method is smoother than derived with the new method. This is caused by the assumption of a constant nonthermal spectral index in the standard method.

- The nonthermal emission from the new method is still more smoothly distributed than the thermal emission. The exponential scale lengths of the nonthermal emission are more than twice as large as those of the thermal emission.

- The standard method overestimates the thermal fraction, especially at the position of giant HII regions. For galactocentric radius $R<7.5 \mathrm{kpc}$, the thermal fractions at $3.6 \mathrm{~cm}$ are $51 \pm 4 \%$ and $63 \pm 5 \%$ from the new and standard methods, respectively.

- For the first time, we have derived a map of the nonthermal spectral index. In the starforming regions, the nonthermal spectrum is relatively flat with an average value of $\alpha_{n}$ of $0.6 \pm 0.1$, the typical spectral index of supernova remnants, but $\alpha_{n}$ increases to $1.2 \pm 0.2$ in the interarm regions and outer parts of the galaxy. This shows that the relativistic electrons lose energy when diffusing from their origin in starforming regions towards interarm regions and the outer parts of the galaxy. The mean spectral index of the nonthermal emission becomes equal to that of the total emission at $R \simeq 4.5 \mathrm{kpc}$. This indicates that the total radio emission is mostly nonthermal at $R>4.5 \mathrm{kpc}$ in M 33, where the spectral index is dominated by synchrotron- (and inverse Compton-) loss processes.

- The north-south asymmetry in the distribution of the thermal emission obtained from the new method is opposite to that from the standard method. The thermal fractions are $47 \pm 5 \%$ and $53 \pm 5 \%$ in the northern and southern halves, respectively.
- Generally, the integrated results from the two methods match each other within the errors, indicating that the assumption of a constant nonthermal spectral index is a proper approximation for "global" studies.

Acknowledgements. We are grateful to P. L. Biermann for valuable and stimulating comments. We thank the staff of the 100-m Effelsberg telescope and the VLA for their assistance with radio observations. F. Tabatabaei was supported for this research through a stipend from the International Max Planck Research school (IMPRS) for Radio and Infrared Astronomy at the Universities of Bonn and Cologne. We acknowledge the M 33 Spitzer collaboration (PI: R. Gehrz) for the MIPS observations.

\section{References}

Andriesse, C. D. 1974, A\&A, 37, 257

Berkhuijsen, E. M. 1982, A\&A, 112, 369

Berkhuijsen, E. M. 1983, A\&A, 127, 395

Berkhuijsen, E. M., \& Klein, U. 1985, in The Milky Way Galaxy, ed.

H. van Woerden, R. J. Allen, \& W. B. Burton, IAU Symp., 106, 431

Berkhuijsen, E. M., Beck, R., \& Hoernes, P. 2003, A\&A, 398, 937

Bicay, M. D., \& Helou, G. 1990, ApJ, 362, 59

Biermann, P. L. 1995, [arXiv: astro-ph/9501030]

Bonanos, A. Z., Stanek, K. Z., Kudritzki, R. P., et al. 2006, ApJ, 652, 313

Boulesteix, J., Courtes, G., Laval, A., Monnet, G., \& Petit, H. 1974, A\&A, 37, 33

Breitschwerdt, D., Dogiel, V. A., \& Völk, H. J. 2002, A\&A, 385, 216

Broadbent, A., Osborne, J. L., \& Haslam, C. G. T. 1989, MNRAS, 237, 381

Buczilowski, U. R. 1988, A\&A, 205, 29

Crockett, N. R., Garnett, D. R., Massey, P., \& Jacoby, G. 2006, ApJ, 637, 741

de Vaucouleurs, G., \& Leach, R. W. 1981, PASP, 93, 190

Deul, E. R., \& van der Hulst, J. M. 1987, A\&AS, 67, 509

Devereux, N., Duric, N., \& Scowen, P. A. 1997, AJ, 113, 236

Dickinson, C., Davies, R. D., \& Davis, R. J. 2003, MNRAS, 341, 369

Dodorico, S. 1978, Mem. Soc. Astr. Ital., 49, 485

Draine, B. T., \& Lee, H. M. 1984, ApJ, 285, 89

Freedman, W. L., Wilson, C. D., \& Madore, B. F. 1991, ApJ, 372, 455

Gordon, S. M., Kirshner, R. P., Duric, N., \& Long, K. S. 1993, ApJ, 418, 743

Gordon, S. M., Kirshner, R. P., Long, K. S., et al. 1998, ApJS, 117, 89

Gordon, S. M., Duric, N., Kirshner, R. P., Goss, W. M., \& Viallefond, F. 1999, ApJS, 120, 247

Helou, G., \& Bicay, M. D. 1993, ApJ, 415, 93

Hinz, J. L., Rieke, G. H., Gordon, K. D., et al. 2004, ApJS, 154, 259

Hippelein, H., Haas, M., Tuffs, R. J., et al. 2003, A\&A, 407, 137

Hoopes, C. G., \& Walterbos, R. A. M. 2000, ApJ, 541, 597

Israel, F. P., \& Kennicutt, R. C. 1980, ApL, 21, 1

Johnson, K. E., Kobulnicky, H. A., Massey, P., \& Conti, P. S. 2001, ApJ, 559, 864

Klein, U., Beck, R., Buczilowski, U. R., \& Wielebinski, R. 1982, A\&A, 108, 176 Klein, U., Wielebinski, R., \& Beck, R. 1984, A\&A, 135, 213

Krügel, E. 2003, The physics of interstellar dust, The physics of interstellar dust, ed. E. Kruegel (IoP Series in astronomy and astrophysics, Bristol, UK: The Institute of Physics)

Kwitter, K. B., \& Aller, L. H. 1981, MNRAS, 195, 939

Magrini, L., Corbelli, E., \& Galli, D. 2007, ArXiv e-prints, 704

Melnick, J., Moles, M., Terlevich, R., \& Garcia-Pelayo, J.-M. 1987, MNRAS, 226, 849

Niklas, S., \& Beck, R. 1997, A\&A, 320, 54

Panagia, N. 1979, Mem. del. Soc. Astron. Ital., 50, 79

Petersen, L., \& Gammelgaard, P. 1997, A\&A, 323, 697

Regan, M. W., \& Vogel, S. N. 1994, ApJ, 434, 536

Rieke, G. H., Young, E. T., Engelbracht, C. W., et al. 2004, ApJS, 154, 25

Schulman, E., \& Bregman, J. N. 1995, ApJ, 441, 568

Shaver, P. A., McGee, R. X., Newton, L. M., Danks, A. C., \& Pottasch, S. R. 1983, MNRAS, 204, 53

Tabatabaei, F., Krause, M., \& Beck, R. 2005, AN, 326, 532

Tabatabaei, F. S., Beck, R., Krause, M., et al. 2007a, A\&A, 466, 509

Tabatabaei, F. S., Krause, M., \& Beck, R. 2007b, A\&A, 472, 785

Valls-Gabaud, D. 1998, Publ. Astr. Soc. Austr, 15, 111

Walsh, W., Beck, R., Thuma, G., et al. 2002, A\&A, 388, 7

Willner, S. P., \& Nelson-Patel, K. 2002, ApJ, 568, 679

Witt, A. N., \& Gordon, K. D. 2000, ApJ, 528, 799

Yang, H., Chu, Y.-H., Skillman, E. D., \& Terlevich, R. 1996, AJ, 112, 146 